\title{
Capacitive Sensors for Whole Body Interaction
}

\author{
Raphael Wimmer \\ University of Munich \\ Amalienstr. 17, 80333 Munich, Germany, \\ raphael.wimmerdifi.lmu.de
}

\begin{abstract}
Capacitive proximity sensors can be used to implement a variety of expressive input devices. They are especially suitable for Whole Body Interaction as they are small, robust, flexible, and can be both worn on the body or embedded into the environment. This chapter discusses technical challenges that arise when using capacitive sensors for tracking human motion, namely sensor shielding and ensuring both low latency and high sensitivity. A custom sensor design and an adaptive moving average filter presented here address these challenges. Two user studies evaluated these sensors as input modalities for different computer games. They found evidence that capacitive sensors offer a friendly but challenging behavior, being easy to learn but hard to master.
\end{abstract}

\section{Introduction}

Using the whole expressivity of human body motion for interacting with intelligent environments or computer systems allows for novel applications and action-ladden exertion games. A first step when implementing such an iteractive system, is choosing an appropriate hand and body tracking technology. Capacitive sensors offer a cheap, robust, and flexible way of prototyping and implementing sensor systems for Whole Body Interaction. However, their capabilities seem to be unknown to many researchers. This chapter presents principles, properties, and prototypes of capacitive sensing for Whole Body Interaction. It has the following structure: Section 2 explains the basic mode of operation of capacitive sensors. Section 3 presents existing input devices using capacitive sensors. Section 4 describes a simple capacitive sensor design that can be used for prototyping capacitive-sensing applications. Section 5 describes technical challenges for capacitive sensing that are especially relevant for human-computer interaction and describes how these can be approached. Section 6 presents learnings from a user study that evaluated different sensor setups as input modalities for computer games. The Conclusion argues why the inherent properties of capacitive sensors make them very well suited for implementing Whole Body Interfaces for games.

\section{Capacitive Sensing}

The term capacitance describes how much electrical charge can be stored between two non-connected conductive objects. These form a capacitor. The capacitance depends primarily on the size of the objects, their distance, and the dielectric properties of objects and insulater between them. A small capacitance always exists between an object 
and its environment.

Capacitive Sensing is a term for a number of different sensing techniques that measure changes in capacitance between a sensor antenna and its surrounding. Smith et al. have characterized three different capacitive sensing techniques: loading mode, transmit mode, and shunt mode $[12,13]$. Loading mode is widely used for touch sensors, while transmit mode and shunt mode are often used for capacitive touchscreens. The following sections focus on loading mode capacitive sensing as it is the simplest and most versatile mode. Several physical effects are neglected for clarity ${ }^{1}$. A more thorough description of capacitive sensing can be found in $[5,12,19]$.

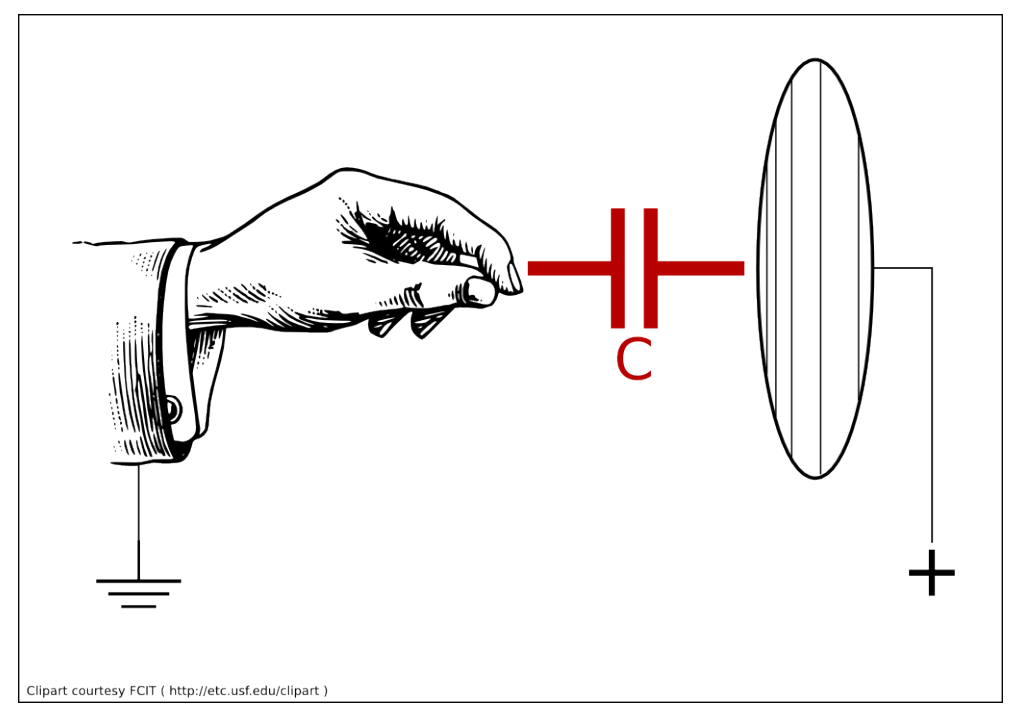

Fig. 1. Basic principle of capacitive sensing. An antenna and a nearby conductive, grounded object form a capacitor. The capacitance of this capacitor is determined by antenna size, object size, distance between both, and material properties. By measuring the capacitance, movement near the antenna can be detected. This can be used for tracking hand or body movement.

A loading mode capacitive sensor measures the capacitance between its antenna and the environment, for example by completely charging this capacitor and discharging it. From the time needed to completely discharge the capacitor, its capacitance can be calculated. In practice the charge/discharge-cycle is often implemented as a resonant circuit. The lower the capacitance at the antenna, the higher the frequency of the circuit. The capacitance greatly depends on the distance between antenna and environment. Thus, bringing part of the environment closer to the antenna increases the capacitance

\footnotetext{
${ }^{1}$ For example, in theory objects need a ground connection for the capacitor to work. However, such a ground connection is provided by capacitive coupling between the object and the environment. Therefore, in practice a conductive ground connection is not needed.
} 
between both. This effect can be used to measure the distance between the antenna and an object near it. In particular, such a sensor can determine the proximity of a human body or hand (Figure 1). Using several sensors one can track a hand or body in three dimensions [18]. However, each object near the antenna contributes to the capacitance that can be measured at the antenna. Therefore, it is impossible to determine how many objects are near the antenna, or how close they are. As capacitive sensors can be used to measure the distance between the antenna and a hand, they allow for easy gestural human-computer interaction .

\section{Related Work}

A variety of sensors are available for tracking human motion. They can be either embedded into the environment or worn by the user. Teixeira et al. conducted a survey of available sensing techniques [16]. One of these are capacitive sensors, sometimes also called electric field sensors. This section provides a short overview of existing research using capacitive sensors for tracking human motion, focusing on novel ways of human-computer interaction..

Probably the first use of capacitive sensors for primitive Whole Body Interaction was in 1907. German physician Max Cremer put a living frog between two capacitor plates. By measuring capacitance changes he could observe the frog's heartbeat [1]. In 1919 Leonid Termen presented the Theremin, a musical instrument using two capacitive sensing antennas for controlling pitch and volume of a tone [2]. The Theremin is played by hand movement near each antenna. It is said to require a great deal of body control as the arms are not mechanically supported and minor trembling of a hand can cause great tone changes. With the Terpsitone, Termen used the same sensing principle for tracking body movement [6]. The sensing circuit is connected to a large metal plate lying on a platform. A dancer on top of the plate would permanently change the capacitance, allowing her to control the pitch of a synthesized tone. Colored lights would light up according to the notes played by the dancer.

Recently, capacitive sensors have been used in a variety of human-computer interfaces. Rekimoto determined hand poses by capturing the wrist shape using a wrist band containing capacitive sensors [9]. Taylor and Bove equipped a baseball with a capacitive sensor matrix which allows to determine how the ball is being held [15]. Valtonen et al. track users by equipping floor tiles with capacitive sensors [17]. By embedding sensors into furniture, one can unobtrusively capture everyday actions [20].

\section{A Simple Capacitive Sensor}

The sensor described here (see Figure 2) is an improved version of the one used in CapToolKit [19]. It has been designed specifically for prototyping interactive systems. The sensor measures the capacitance between the attached antenna and the environment. This is done by an LMC555 timer IC. It outputs a signal with a frequency between 0 and $2 \mathrm{MHz}$, depending on the measured capacitance. The sensor is intended to be connected to a microcontroller that continuously measures the frequency and filters the resulting signal. 

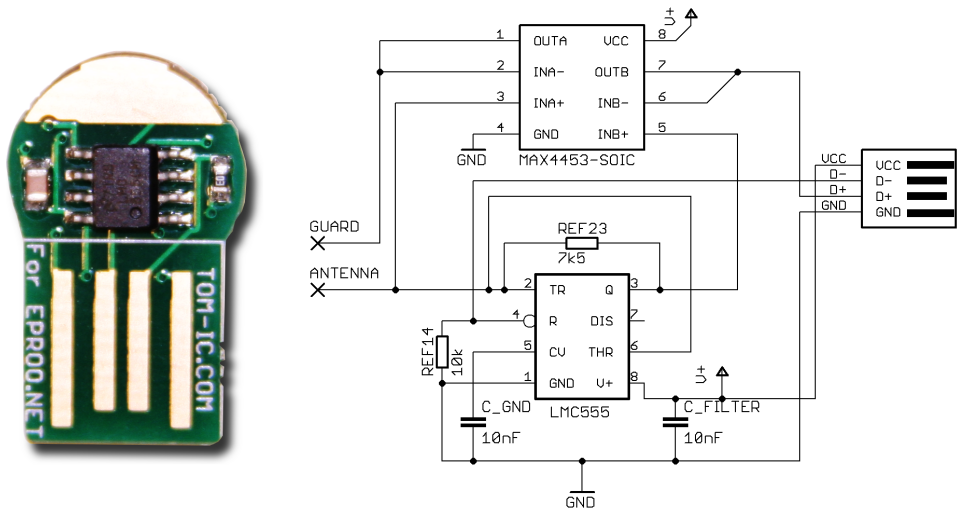

Fig. 2. Photo and schematic of a simple capacitive sensor. An LMC555 timer IC converts the capacitance between an antenna and the environment to a high-frequency digital signal. A MAX4453 dual-op amp (on the back of the circuit board) is used to shield the antenna from unwanted capacitive coupling.

A common requirement for a capacitive sensor is that it should only sense objects in a certain directions. Generally, the antenna of a capacitive sensor is omnidirectional. Usually, this is not wanted for two reasons: Firstly, any objects moving near the antenna affect the sensor readings. For example, people passing near an installation using capacitive sensors might interfere with its intended operation. Secondly, antennas that are placed on other objects - like a wall-mounted or a body-worn sensor - get electrically tainted by these objects. This results in decreased sensor range and resolution. A capacitive sensor mounted on a user's chest would be saturated by the capacitance to the chest. Thus, a hand moving near the sensor would only minimally affect the overall capacitance.

Therefore, capacitive sensor antennas should be shielded from the environment in one or more directions. However, a grounded electrode - as normally used for shielding electronics - would form a capacitor together with the antenna, even decreasing the sensor's range. Instead, the shield electrode needs to be actively kept at the same electrical potential as the antenna. This has to be done without galvanically connecting both, however. In order to distinguish them from passive shields, such active shields are called guards or guard electrodes. Commonly, antenna and guard are connected to a sensor chip or microcontroller by cables. This requires the antenna cable to be guarded, too. Our sensor gets directly soldered to both antenna and guard electrode, avoiding cables that would need to be guarded. As the sensor is very small $(20 \times 10$ $\mathrm{mm}$ ), the antenna/sensor-combination is still easily embeddable. The sensor's connector is compatible to USB-A plugs. This allows to use off-the-shelf USB extension cables for connecting the sensor to the sensing board. These are cheap, capable of transmitting high-frequency signals, and available in a multitude of lengths and types. 
Placing sensor and antenna as close together as possible has another advantage: the conversion from (analog) capacitance to (digital) frequency happens as soon as possible. This leaves little room for electric fields and other noise sources to affect the sensor reading. The frequency-encoded signal can be transferred over long distances without degradation.

For coupling antenna electrode and guard electrode, a voltage follower circuit can be used [11]. Such a circuit ensures that the voltage at its output is always kept at the same level as its input. Unlike a direct connection, it does not propagate capacitance changes at the output to the input. The MAX4453 integrated circuit (IC) combines two rail-to-rail ${ }^{2}$ operational amplifiers (op-amps) in one chip package. One op-amp drives the guard electrode while the other capacitively insulates the sensor's output line. This is necessary to protect the sensor readings from capacitance changes near the microcontroller board or the host PC.

\section{Signal Processing}

Capacitive sensors suffer from two sources of errors as illustrated in Figure 3:

Random noise is inherent to all analog sensors. For touch sensing, this noise is usually several factors lower than the capacitance change caused by touching the sensors. For proximity sensing, however, the signal-to-noise ratio (SNR) of a sensor determines its range. With increasing distance between sensor and object, the signal amplitude decreases quadratically. As the noise level stays the same, the SNR quickly decreases. Once the signal level falls below the noise level, the object can no longer be detected. A simple moving average filter is very effective against such random noise ([14]. It attenuates signal components with high frequency (usually noise) and leaves signal components with low frequency (slow movement near the sensor) intact. The filter adds the last $n$ samples obtained from the sensor and divides the sum by $n$. The lower the SNR, the more samples need to be averaged in order to eleminate random noise. However, the moving average filter inevitably introduces latency, delaying and attenuating the effects of capacitance changes.. The more sensitive a sensor needs to be, the more samples need to be averaged, and the greater the latency. However, for human-computer interaction, and especially for computer games, a latency of significantly less than 100 $\mathrm{ms}$ is desirable.

Sensor drift is especially problematic for capacitive sensors. These are very susceptible to changes in environmental humidity and temperature. Such gradually changing parameters can hide important events or generate fake events. For example, once started, a sensor might heat up minimally. Such minimal temperature changes lead to increasing sensor readings. These readings can be misinterpreted as a slow movement of an object towards the sensor. Usually the sensor drift is very slow so that it can be clearly distinguished from movement events. However, sometimes the measured capacitance quickly changes due to changes in the environment. For example, a conductive object might have been placed near the sensor. Also, the sensor antenna might have been deformed, resulting in a significantly different sensor reading. Ususally, such changes should be filtered out.

\footnotetext{
${ }^{2}$ Rail-to-Rail means that the output voltage can reach the full range of the input voltage.
} 

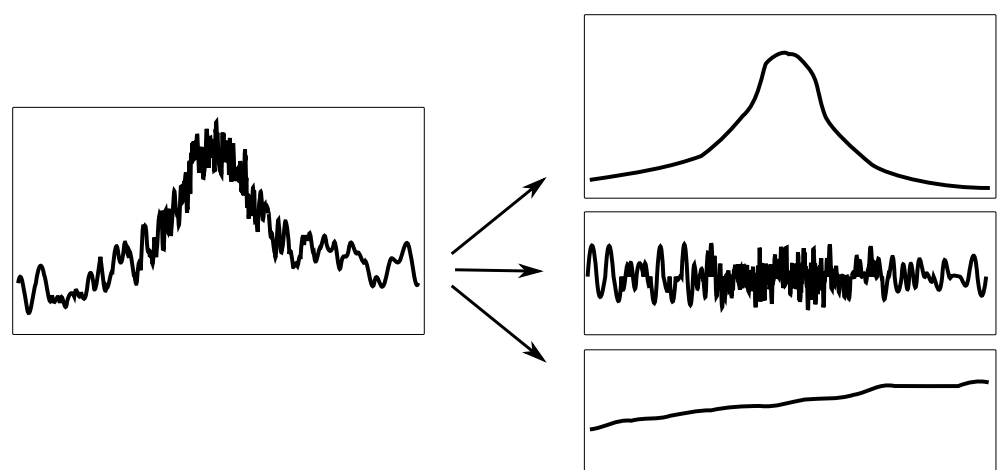

Fig. 3. Components of capacitive sensor readings: signal caused by a person passing the sensor (top right), random noise of the electrical circuit (center right), and drift due to changes in temperature or humidity (bottom right).

CapToolKit [19] aggressively filters out noise while still offering low latency interaction. To this end, we utilize the speed-accuracy tradeoff inherent to human motion: the more precise a movement needs to be, the slower it has to be performed - and vice versa[8]. Therefore, the sensor needs to be either fast or sensitive, not both at a time. To this end, the microcontroller that transmits sensor readings to a computer applies an adaptive moving average (AMA) filter to the raw sensor data. The AMA is a moving average filter that adjusts the number of samples used for averaging based on the current signal properties. Initially, the sensor uses a 16 sample buffer for averaging. This almost completely eliminates random noise. Very small movement - resulting in very small capacitance changes - can be reliably tracked in this way. However, this also introduces a latency of several hundred milliseconds. For slow movement this is no problem, for rapid movement such a latency is not acceptable. Therefore, the AMA continuously observes the unfiltered signal. If there is a change in the signal that is much larger than random noise, the filter assumes a large, fast movement nearby the sensor. The filter then immediately reduces the number of samples used for averaging. This decreases latency. The larger the sudden change in the signal, the less averaging will be applied to the signal. Once the signal changes less rapidly, the number of samples used for averaging is increased again.

When no movement is detected, the sensor is automatically recalibrated at regular intervals, compensating for temperature and humidity drift. In order to not mistakenly compensate small movements, the recalibration only takes place if the sensor reading has constantly and evenly risen or fallen for some time.

\section{Game Controllers built from Capacitive Sensors}

We conducted a first user study in order to learn more about the suitability of capacitive sensors for controlling different types of computer games. In this study, 13 participants ( 6 female, mostly students, on average 25 years old) had to play three different games. 
The games' concepts and graphics were kept very simple in order to reduce confounding variables and provide a coherent feel across the three games.
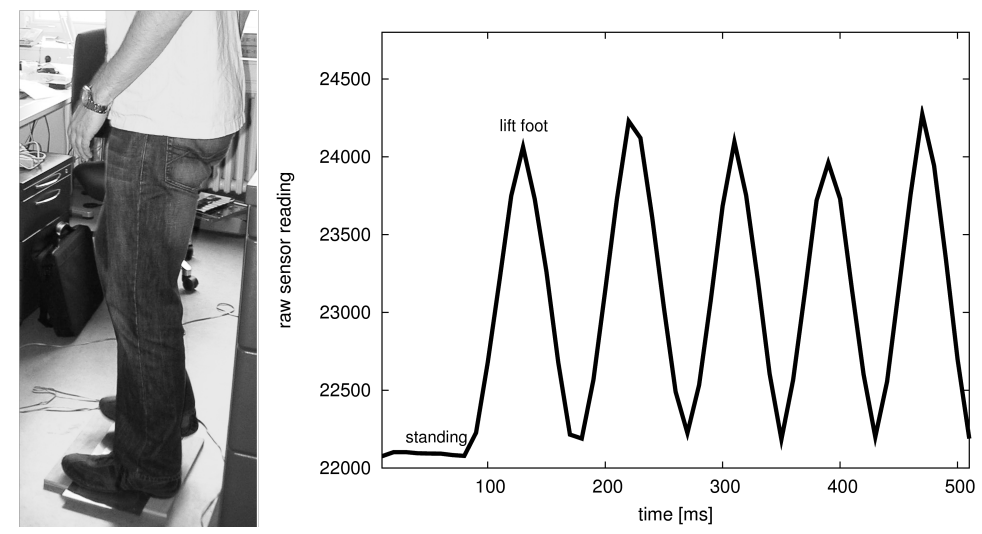

Fig. 4. A simple Whole Body Interface that was prototyped using capacitive sensors. The user controls a ball by walking on top of a metal plate that acts as the sensor antenna. The sensor readings allow to determine pace and amplitude of the stride.

The first game, MoveBall, required fine motor skills. Four capacitive sensor strips were placed at on a tabletop, forming a square. A computer screen showed a 2D, topview arena with a randomly placed ball and a randomly placed hole. By approaching one of the sensors with the hand, the user could move the ball in one of the the four directions. In each round it was counted how many times the user could move the ball into the hole within one minute. The second game, HitBall, required gross motor skills. The user stood on a large sensor plate. Two antennas were placed at arms length to his/her left and right. A red circle appeared randomly at the top, left, or right edge of the screen. Depending on its position the user had to reach out towards the left or right antenna, or jump. In each round it was counted how many times the user "hit" the correct target. The third game, WalkBall (see Figure 4), required simultaneous movements. The sensor layout was the same as with HitBall. The goal was the same as with MoveBall, moving a ball into a hole. The user could move the ball forward by walking in place. By leaning towards the left or right antenna, the user could change the ball's direction. In each round it was counted how many times the user could move the ball into the hole within one minute.

The order of the games was counterbalanced across the participants. The participants first played each game once using the cursor keys of a keyboard in order to familiarize themselves with the game's concept. Afterwards, they played three rounds of each game, answering a questionnaire between rounds.

There was no statistically significant learning effect regarding the scores for HitBall and WalkBall. However, we observed a learning effect $(\mathrm{p}<0.002)$ for MoveBall which exercises fine motor skills. Obviously, simple, gross movements do not require much 
training - precise control does. Participants didn't generally feel more exerted using capacitive sensor controls for the three simple games than using the keyboard. Only for the HitBall game capacitive sensor controls required significantly higher exertion $(p<0.05)$. Keyboard control was significantly less challenging than capacitive sensor control for all of the simple games. This shows both in higher game scores $(\mathrm{p}<0.01$ for all games) and the answers to the questionnaire: Participants felt more challenged using the capacitive sensor controls $((\mathrm{p}<0.05$, except for WalkBall, where participants felt challenged using the keyboard, too). Despite (or because of) the greater challenge, participants preferred capacitive sensor controls over the keyboard for all three simple games $(\mathrm{p}<0.05)$. Capacitive sensor controls were especially preferred for HitBall, which requires rapid physical action.

These findings suggested that capacitive sensors are easy to learn but precise control is challenging. This is in fact a wanted behavior for games.

Myers found 'challenge' to be, "the most preferred characteristic of a favorite game" [7]. In his book "A Theory of Fun", game designer Raph Koster describes one key principle of games:

When you're playing a game, you'll only play it until you master the pattern. Once you've mastered it the game becomes boring. [3]

Thus, a game should be complex and difficult enough to challenge the player. A game controller can support this behavior by being hard to master. On the other hand, it needs to be intuitive and easy to learn. In this context, "learning" can be seen as understanding the basic capabilities and the mapping of the controller, "mastering" as getting the controller to do what the player wants.

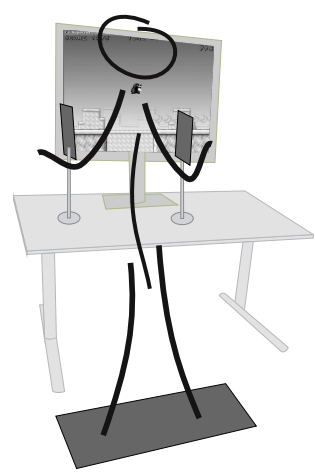

Fig. 5. In a second study users played a jump'n'run video game using capacitive sensors. By approaching one of the two antennas mounted in front of the user, he/she could move a penguin left or right. When the user jumped, the penguin would jump accordingly.

To investigate whether capacitive sensors were indeed "easy to learn but hard to master", we conducted a second study with 16 participants ( 7 female, mostly students, on average 24 years old). Participants had to play a jump'n'run video game, SuperTux. 
They controlled a small penguin using three capacitive sensors. Two antennas mounted in front of the user controlled left/right movement while an antenna plate on the ground detected when the user was jumping, making the penguing jump, too (see Figure 5). Again, the users first played the game using a keybaord to familiarize themselves with the game. Afterwards, they played using the capacitive sensors. In both cases the users could play as long as they wanted. On average, the game was played for 1.75 minutes using the keyboard and for 3.94 minutes using the capacitive sensors. Afterwards, the participants answered a questionnaire.

On 5 point Likert scale all participants answered that the capacitive sensors reproduced their movements accurately or very accurately. 13 of the 16 participants found the capacitive sensor controls to be challenging. Those participants who enjoyed the capacitive sensor controls more than the keyboard (12 of 16) also found them more challenging $(\mathrm{p}<0.01)$.

We asked participants how long it took them to understand the capacitive sensor controls and how long it took them to actually master them. Learning and mastering were defined as above. Most participants understood the controls quickly or very quickly but mastered it only slowly. Figure 6 shows the distributions. Obviously, participants took significantly less time to understand the controls than to master them $(\mathrm{p}<0.001)$. Unfortunately, we asked slightly different questions about the keyboard control. Therefore, it is not clear whether this difference also hold true for keyboard control.

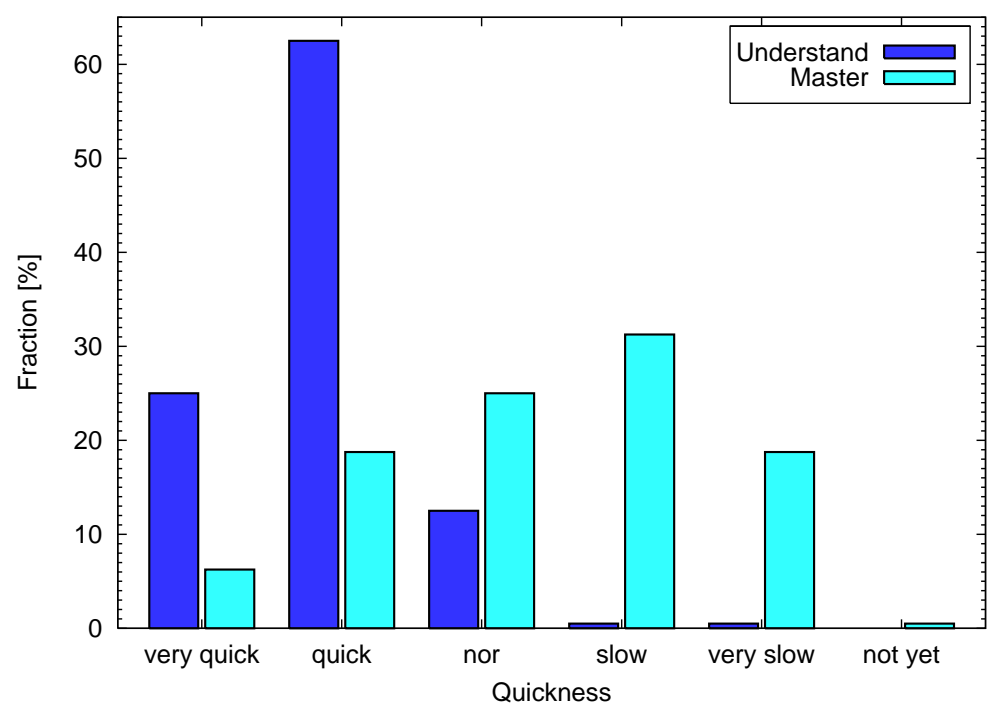

Fig. 6. Participants playing a jump'n'run game using capacitive sensors took significantly less time to understand the controls than to master them 
We found no significant correlation between how often people play sports and how challenging or exertive they rated the capacitive sensor controls. However, people who play sports at least once a week would like to use capacitive sensor controls again. People who play sports less often are undecided. Using capacitive sensor controls participants enjoyed playing the game at least as much as when using the keyboard. If participants already had played an exertion game before (mostly Wii games) they prefered capacitive sensor controls over the keyboard $(\mathrm{p}<0.001)$. They enjoyed these controls more than the keyboard $(\mathrm{p}<0.02)$ and would use them again $(\mathrm{p}<0.05)$.

Overall, these studies provide some evidence that game controllers using capacitive sensors can add an additional layer of complexity to game. While novice players have little trouble understanding the mapping between their movements and the game's reaction, ample time is needed for actually mastering the controls, giving skilled players an advantage.

\section{Conclusion}

Due to some unique properties, capacitive sensors are especially suited for Whole Body Interfaces, especially for game controllers:

Robust Hardware. Capacitive sensors have no moving or fragile parts. The sensor itself is a small circuit board that can be hidden almost everywhere. The antenna can be made of different materials, e.g. a sturdy metal plate or a flexible fabric, depending on the application. Thus the sensors can be easily embedded into installments, play objects, or clothes. We have also embedded wireless sensors in small tangible interfaces, using Bluetooth for sending sensor data to a host PC.

Flexible Placement and Layout. Capacitive sensors are very versatile. The onedimensional, continuous sensor output can be directly used as an input parameter for a game. The sensitivity and direction of the sensor is determined by electrode size, shape and placement. Sensors can be customized for an application, so that they are sensitive only to specific movements. For example, the force of a punch or touch can be measured by glueing a piece of foam on top of the antenna [4]. The stronger the punch, the more the foam gets compressed, and the closer the user's hand gets to the electrode. Thus, the sensor effectively measures the remaining distance between fist and electrode. The one-dimensional, continuous sensor output can be directly used as an input parameter for a game.

Capacitive sensors may also be used for tracking more complex motion. The capacitive coupling between user and ground continuously changes while walking. By putting a sensor below the user's feet or by attaching a sensor to the user[10], walking motions can be detected and quantified.

Friendly but Challenging Behavior. As capacitive sensors generate data of very low complexity, they allow for a straightforward mapping from sensor output to input event. A user can quickly grasp the correlation between his/her movements and the system's reaction. Thus the barrier to entry of such an interface can be very low.

However, the many influencing factors determining a sensor reading also introduce higher complexity. While new users may quickly learn the basic usage of such an input device, they may needs lots of time in order to master the device. Accidental obser- 
vations or attempts to fudge the device may open up entirely new game tactics. For example, a user may learn from which direction to approach a sensor in order to remain undetected or effect a higher sensor reading. Requiring extra effort in order to master a game corresponds with many physical game 'interfaces', like bats and balls.

In conclusion, capacitive sensors are a simple and versatile sensor type that is especially suited for Whole Body Interaction. Off-the-shelf sensors and simple custom-built sensors allow tracking of body movement, touch, and pressure. Additionally, capacitive sensors can also measure a variety of other properties and actions like finger position, fluid level, or proximity to other objects. Some examples for such interfaces might be: sturdy, foam-covered sensor bars, which the user has to hit at the right places in order to destroy enemies attacking his avatar on the computer screen. Balls may determine, if they are held by one or more persons, or lying on the ground. An game like 'Dance Dance Revolution' might not only track feet movement, but the whole body. The filtering techniques described in this chapter can also be applied to other domains where high precision and low latency are required.

Acknowledgement: The user studies were planned, conducted, and analyzed by Annette Reiter, a graduate student I supervised.

\section{References}

1. Cremer, M.: Ueber die Registrierung mechanischer Vorgänge auf electrischem Wege, speziell mit Hilfe des Saitengalvanometers und Saitenelectrometers. Münchner Medizinische Wochenschrift 54, 1629-1630 (1907)

2. Glinsky, A.V.: The Theremin in the Emergence of Electronic Music. Ph.D. thesis, New York University (1992)

3. Koster, R.: A Theory of Fun for Game Design. Paraglyph Press (2004)

4. Lee, C.H., Hu, Y., Selker, T.: iSphere: a Proximity-based 3D Input Interface. In: Proceedings of CAAD Futures 2005 (2005). URL http://web.media.mit.edu/ jackylee/publication/209J.pdf

5. Lion Precision: Capacitive Sensor Operation and Optimization. Tech. rep. (2006). URL http://www.lionprecision.com/tech-library/technotes/cap-0020-sensor-theory.html

6. Mason, C.: Terpsitone. A New Electronic Novelty. Radio Craft 335 (1936)

7. Myers, D.: A q-study of game player aesthetics. Simul. Gaming 21(4), 375-396 (1990). DOI http://dx.doi.org/10.1177/104687819002100403. URL http://dx.doi.org/http://dx.doi.org/10.1177/104687819002100403

8. Plamondon, R., Alimi, a.M.: Speed/accuracy trade-offs in target-directed movements. Behavioral and Brain Sciences 20(2), 279-303; discussion 303-49 (1997). URL http://www.ncbi.nlm.nih.gov/pubmed/10096999

9. Rekimoto, J.: Gesturewrist and gesturepad: Unobtrusive wearable interaction devices (2001). URL citeseer.ist.psu.edu/rekimoto01gesturewrist.html

10. Rekimoto, J., Wang, H.: Sensing gamepad: electrostatic potential sensing for enhancing entertainment oriented interactions. In: CHI '04, pp. 1457-1460. ACM Press, New York, NY, USA (2004). DOI http://doi.acm.org/10.1145/985921.986089. URL http://dx.doi.org/http://doi.acm.org/10.1145/985921.986089

11. Reverter, F., Li, X., Meijer, G.: Stability and accuracy of active shielding for grounded capacitive sensors. Measurement Science and Technology 17, 2884 (2006)

12. Smith, J.: Electric Field Imaging. Ph.D. thesis, MASSACHUSETTS INSTITUTE OF TECHNOLOGY (1999). URL http://web.media.mit.edu/ jrs/phd.pdf 
13. Smith, J., White, T., Dodge: Electric Field Sensing For Graphical Interfaces. Computer Graphics and Applications 18(3), 54-61 (1998). URL http://www.media.mit.edu/physics/publications/papers/98.02.CGA_Final.pdf

14. Smith, S.: Digital signal processing: a practical guide for engineers and scientists, p. $278 \mathrm{f}$. Newnes (2003)

15. Taylor, B.T., Bove, M.V.: Graspables: grasp-recognition as a user interface. In: In Proc. CHI '09, pp. 917-926. ACM, New York, NY, USA (2009). DOI 10.1145/1518701.1518842. URL http://dx.doi.org/10.1145/1518701.1518842

16. Teixeira, T., Dublon, G., Savvides, A.: A Survey of Human-Sensing: Methods for Detecting Presence, Count, Location, Track, and Identity

17. Valtonen, M., Maentausta, J., Vanhala, J.: Tiletrack: Capacitive human tracking using floor tiles. In: PERCOM '09: Proceedings of the 2009 IEEE International Conference on Pervasive Computing and Communications, pp. 1-10. IEEE Computer Society, Washington, DC, USA (2009). DOI http://dx.doi.org/10.1109/PERCOM.2009.4912749

18. Wimmer, R., Holleis, P., Kranz, M., Schmidt, A.: Thracker - Using Capacitive Sensing for Gesture Recognition. ICDCSW 0, 64 (2006). DOI http://doi.ieeecomputersociety.org/10.1109/ICDCSW.2006.109.

URL http://dx.doi.org/http://doi.ieeecomputersociety.org/10.1109/ICDCSW.2006.109

19. Wimmer, R., Kranz, M., Boring, S., Schmidt, A.: A Capacitive Sensing Toolkit for Pervasive Activity Detection and Recognition. In: PerCom '07 (2007)

20. Wimmer, R., Kranz, M., Boring, S., Schmidt, A.: CapTable and CapShelf-Unobtrusive Activity Recognition Using Networked Capacitive Sensors. In: Fourth International Conference on Networked Sensing Systems, 2007. INSS'07, pp. 85-88 (2007) 